\title{
RELAÇÃO ENTRE A BIOMASSA DA VEGETAÇÃO CAMPESTRE NATIVA E DADOS DE SENSORIAMENTO REMOTO ORBITAL
}

\author{
Carline Biasoli Trentin* \\ Universidade Federal do Mato Grosso
}

\author{
Aline Biasoli Trentin** \\ Universidade Federal do Pampa
}

\author{
Dejanira Luderitz Saldanha*** \\ Universidade Federal do Rio Grande do Sul
}

Resumo: As variações da resposta espectral da vegetação estão diretamente relacionadas com a quantidade de biomassa aérea produzida (estocada) além de outros pigmentos. 0 objetivo deste trabalho foi estimar a biomassa aérea da vegetação através de uma relação entre a biomassa da parte aérea da vegetação campestre nativa coletada em campo e dados de sensoriamento remoto orbital, considerando as estações quente e fria do ano. Para estimar a biomassa aérea da vegetação campestre nativa a partir de dados espectrais, foram analisados os dados de quantidade de biomassa aérea instantânea coletada em campo durante o período de 2012 a 2014 e os dados da resposta espectral da vegetação (medidos pelo sensor MODIS). A partir da elaboração de um perfil temporal dos dados e um gráfico de dispersão entre os valores de biomassa aérea e dados espectrais, foram realizadas análises de correlação e análise de regressão linear, verificando a relação existente entre estes dois conjuntos de dados. Desta forma, observou-se que a vegetação campestre nativa do bioma Pampa tem um comportamento sazonal bem definido, com período de crescimento das espécies nos meses quentes do ano. Foi verificada uma relação direta entre a biomassa aérea e os índices EVI e NDVI e a banda espectral do NIR, com maiores valores no verão e menores no inverno. O coeficiente de correlação foi significativo para as bandas do azul, vermelho, NIR, EVI e NDVI. Desta forma, o modelo resultante demonstrou a aplicabilidade das imagens MODIS para a estimativa da biomassa aérea da vegetação campestre.

Palavras-chave: Resposta espectral. Sensor MODIS. Bioma Pampa.

\section{RELATIONSHIP BETWEEN BIOMASS OF NATIVE GRASSLANDS AND REMOTE SENSING DATA}

Abstract: Variations of spectral response are directly related to the amount of aboveground biomass vegetation (stored) in addition to other pigments. We estimate the aboveground biomass vegetation through a relationship between aboveground biomass vegetation in grassland native collected in the field and remote sensing data in the hot and cold seasons of the period from 2012 to 2014 . To estimate the biomass of grassland native air from spectral data, the data were related to amount of instant air field collected biomass during the period 2012 to 2014 versus the spectral response of vegetation data (measured by MODIS sensor). From the development of a temporal profile of data and a scatter plot between aboveground biomass values and spectral data, conducted analyses of correlation and linear regression analysis, noting the relationship between these two sets of data. In this way, it was observed a relationship between aboveground biomass and the indices EVI and NDVI and NIR spectral band, with highest values in summer and lower in winter. The correlation coefficient was significant for the bands of blue, red, NIR, EVI and NDVI. In this way, the resulting model demonstrated the applicability of MODIS images for the estimation of aboveground biomass of grassland vegetation.

Keywords: Spectral response. MODIS sensor. Pampa Biome.

\section{RELACIONES ENTRE LA BIOMASA DE LA VEGETACIÓN CAMPESTRE NATIVA Y DATOS DE DETECCIÓN REMOTA ORBITAL}

Resumen: Las variaciones de la respuesta espectral de la vegetación están directamente relacionadas a la cantidad de biomasa aérea producida (estoqueada) además de otros pigmentos. El objetivo de este trabajo fue estimar la biomasa aérea de la vegetación a través de una relación entre la biomasa de la parte aérea de la vegetación campestre nativa colectada en el campo y datos de detección remota orbital, considerando las estaciones caliente y fría del año. Para estimar la biomasa aérea de la vegetación campestre nativa a partir de datos espectrales, fueron analisados los datos de cuantidad de biomasa aérea instantánea colectada en el campo durante el período de 2012 a 2014 y los datos de la respuesta espectral de la vegetación (medidos por el sensor MODIS). A partir de la elaboración de un perfil temporal de los datos y un gráfico de dispersión entre los valores de biomasa aérea y datos espectrales, fueron realizados análisis de correlación y análisis de regresión linear, verificando la relación existente entre estos dos conjuntos de datos. De esta manera, se observó que la vegetación campestre nativa del bioma Pampa tiene un comportamiento estacional bien definido, con período de crecimento de las especies en los meses calientes del año. Fue verificada una relación directa entre la biomasa aérea y los índices EVI y NDVI y la banda espectral del NIR, con mayores valores en el verano y menores en el invierno. El coeficiente de correlacción fue significativo para las bandas del azul, rojo, NIR, EVI y NDVI. Así, el modelo resultante demostró la aplicabilidad de las imagenes MODIS para la estimativa de la biomasa aérea de la vegetación campestre.

Palabras clave: Respuesta espectral. Sensor MODIS. Bioma Pampa.

*Universidade Federal do Mato Grosso, Doutora em Sensoriamento Remoto. Instituto de Ciências Humanas e Sociais - Curso de Geografia, Av. Valdon Varjão, 6390 - Campus Universitário do Araguaia, Barra do Garças/MT-Brasil, CEP 78600-000. ORCID: https://orcid.org/0000-0002-7678-1320. E-mail:<carlinebtrentin@gmail.com

*Universidade Federal do Pampa, Doutora em Geografia. Avenida Antônio Trilha, 1847, Campus São Gabriel, São Gabriel/RS-Brasil, CEP 97300-162. ORCID: https://orcid. org/0000-0001-9747-9289. E-mail: abtrentin@gmail.com

**Universidade Federal do Rio Grande do Sul, Doutora em Geociências. Instituto de Geociências - Departamento de Geodésica, Av. Bento Gonçalves, 9500 - Bloco I, Prédio

43136 - Bairro Agronomia, Porto Alegre/RS, Brasil, CEP 91500-000.E-mail: dejanira.saldanha@ufrgs.br 


\section{Introdução}

A composição florística da vegetação define a alocação de biomassa e a sua produtividade (Silveira et al., 2005, p. 583; Fonseca et al., 2006, p. 657). Portanto, o monitoramento da biomassa ao longo do tempo pode fornecer importantes informações sobre a estabilidade do ecossistema natural.

A quantificação da biomassa em ecossistemas naturais pode ser estimada a partir de dados de campo, dados espectrais ou por modelos estatísticos (Paruelo et al., 2000, p. 190; Wolf et al., 2011, p. 1.140). O uso de sensoriamento remoto para o monitoramento da biomassa aérea da vegetação campestre é feito através de modelos estatísticos que relacionam a resposta espectral da vegetação com a biomassa aérea disponível no momento da passagem do satélite (Bork et al., 1999, p. 249; Tueller, 2001, p. 88; Bombelli et al., 2009, p. 2.196).

As imagens de satélite possibilitam monitorar continuamente os processos dinâmicos da vegetação (Menzel et al., 2001, p. 659). Nos últimos anos, estudos utilizando imagens de satélite com o objetivo de monitorar a biomassa da vegetação em diferentes formações vegetais tiveram um aumento na sua importância devido ao contexto de mudanças climáticas, as quais podem alterar o início das fases fenológicas, tais como o período de crescimento e a distribuição da vegetação.
A quantidade de biomassa da vegetação está correlacionada com os pigmentos fotossintetizantes e espaços intercelulares das folhas que interagem com a radiação eletromagnética, provocando alterações na resposta espectral nas bandas do visível e infravermelho próximo (Jensen, 2009, p. 379). Assim, as variações da resposta espectral estão diretamente relacionadas com a quantidade de biomassa aérea da vegetação.

0 objetivo deste estudo foi estimar a biomassa aérea da vegetação campestre nativa por meio de uma relação entre a biomassa da parte aérea da vegetação coletada em campo e dados de sensoriamento remoto orbital nas estações quente e fria do ano (verão e inverno, respectivamente).

\section{Área de estudo}

O bioma Pampa está localizado em regiões na América do Sul como na Argentina, Brasil e Uruguai. No Brasil se restringe ao estado do Rio Grande do Sul, ocupando originalmente $63 \%$ do território do estado. 0 relevo é caracterizado por planícies, suavemente onduladas a onduladas. O clima é subtropical e apresenta grande variação sazonal com verão quente e seco e inverno frio e úmido. De acordo com a classificação de Köppen, é considerado como Cfa (clima temperado úmido com verão quente) (Overbeck et al., 2007, p. 1.074).

Figura 1 - Mapa de localização da área de estudo.

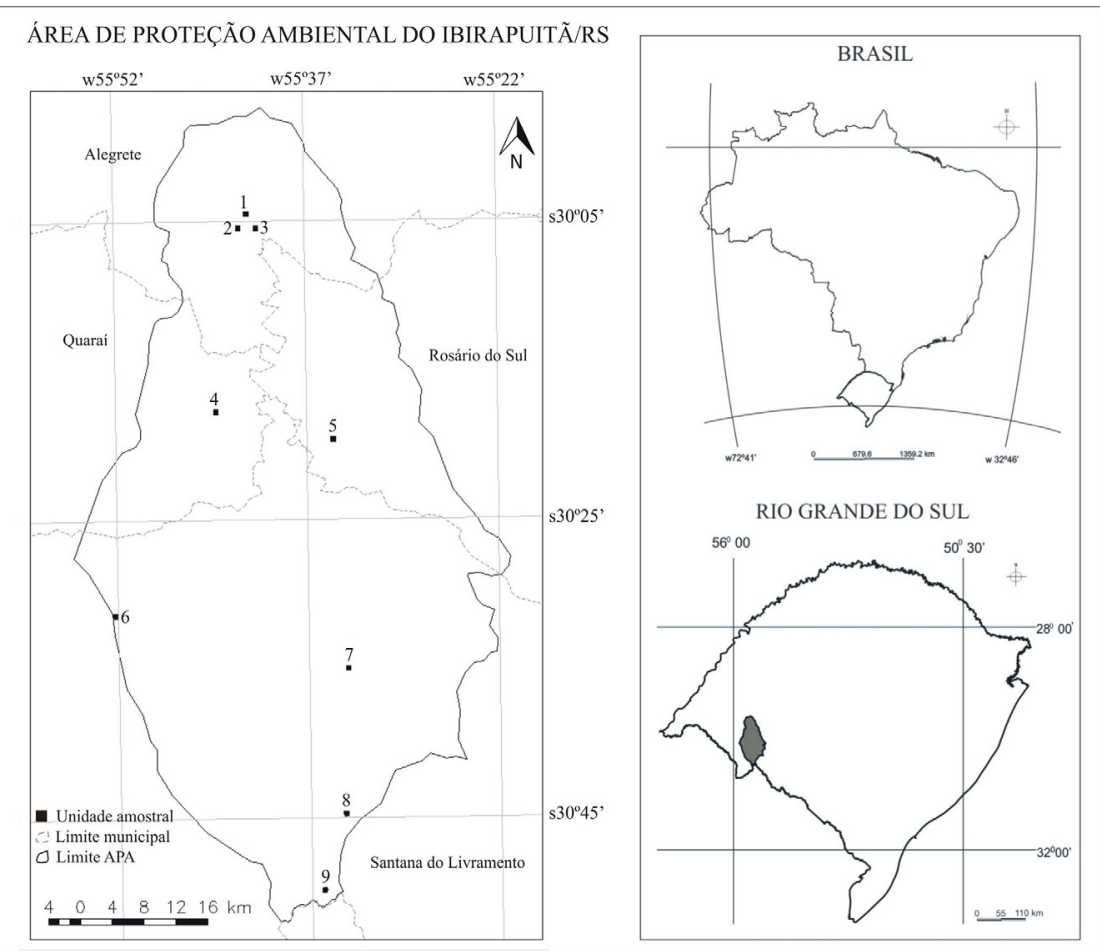

Fonte: Elaborado pela autora. 
Possui vegetação caracterizada por plantas herbáceas do tipo C3 e C4 adaptadas à transição do clima subtropical para o temperado (Boldrini, 2009, p. 67). O período de crescimento das espécies de gramíneas predominantes no bioma Pampa (C4) se inicia na primavera e vai até o outono, período com maior disponibilidade de radiação e temperaturas mais altas, resultando em uma maior produção de biomassa nas estações quentes do ano (Heringer; Jacques, 2002, p. 602).

Neste trabalho foi definido como local de estudo a Área de Proteção Ambiental (APA) do Ibirapuitã (Figura 1), localizada no bioma Pampa, na porção sul-sudoeste no estado do Rio Grande do Sul/Brasil, com uma área de 318.767 hectares. É uma unidade de conservação de uso direto, conforme Decreto de Lei Federal n. 529 de 20 de maio de 1992, gerenciada pelo IBAMA (Instituto Brasileiro do Meio Ambiente e dos Recursos Naturais Renováveis), sendo a única unidade de conservação federal representante do Bioma Pampa no Brasil.

\section{Material e métodos}

Para estimar a biomassa aérea da vegetação campestre nativa a partir de dados espectrais, foram comparados os dados de quantidade de biomassa aérea coletada em campo e da resposta espectral da vegetação (medidos pelo sensor MODIS). As amostras de biomassa aérea da vegetação foram coletadas em campo no período de verão (janeiro de 2012 e 2013, fevereiro de 2014) e inverno (julho de 2012 e 2013). As épocas foram escolhidas devido ao maior e menor acúmulo de biomassa da vegetação do bioma Pampa (Carvalho; Maraschin; Nabinger, 1998, p. 6), respectivamente, visando um entendimento da dinâmica sazonal da vegetação associada às variações climáticas intra-anuais existentes na região.

Foram definidas nove unidades amostrais, localizadas no interior da APA (Figura 1), observando as variações de tipos de solo presentes na área de estudo (Neossolo, Argissolo e Vertissolo). As coletas foram realizadas em cinco pontos aleatórios dentro de cada unidade amostral, sendo que o resultado da amostra corresponde à média aritmética destes cinco pontos.

A área da amostra foi delimitada através do "método do quadrado", utilizando um quadrado de barra de ferro de $50 \mathrm{~cm} \times 50 \mathrm{~cm}$. A parte aérea da vegetação foi coletada utilizando uma tesoura, cortando toda a vegetação rente ao solo no interior desse quadrado (Salman; Soares; Canesin, 2006, p. 2).

As imagens correspondentes às bandas do azul, vermelho, infravermelho próximo (NIR) e infravermelho médio (MIR), NDVI (Normalized Difference Vegetation Index) e EVI (Enhanced Vegetation Index) do sensor MODIS/Terra produto MOD13Q1 têm resolução espacial de $250 \mathrm{~m}$ e resultam de um mosaico de pixels escolhidos entre as imagens diárias em um tempo de 16 dias, objetivando extrair pixels com melhor qualidade (sem interferência de nuvens e rú́dos). Consideraram-se as imagens MODIS correspondentes aos dias julianos 033 (verão) e 209 (inverno) para os anos de 2012 e 2013 e 049 (verão) para o ano de 2014, com o propósito de analisar os dados orbitais a partir do mesmo período da coleta em campo.

Os valores da resposta espectral das bandas do sensor MODIS foram extraídos para cada um dos cinco pontos aleatórios (definidos nas coletas de campo), dentro das unidades amostrais, sendo que cada pixel representa uma amostra. Assim, o valor espectral da unidade amostral corresponde à média dos cinco pixels (mesma localização das amostras de campo). Para a extração automática dos valores da resposta espectral das bandas do sensor MODIS de cada uma das amostras, utilizou-se o módulo "extração de estatística de imagem por polígono" disponível no software SPRING (Sistema de Processamento de Informações Georreferenciadas), versão 5.1.8, disponibilizado pela Divisão de Processamento de Imagens/Instituto Nacional de Pesquisas Espaciais (DPI/INPE).

Foi gerado um perfil temporal para os valores de biomassa aérea e para os dados espectrais, considerando a média aritmética das nove unidades amostrais, visando observar o comportamento geral da vegetação campestre no interior da APA. Também foi observada a dispersão entre os valores e realizadas análises de correlação entre os dados de biomassa e a resposta espectral de cada uma das bandas do sensor MODIS (produto MOD13Q1). Posteriormente fez-se uma análise de regressão linear, tendo como variável dependente a biomassa da vegetação medida pelo sensor, verificando a relação existente entre estes dois conjuntos de dados.

Para fins de comparação entre os valores de biomassa estimada e as variáveis meteorológicas, foram coletados dados mensais de precipitação pluvial e temperatura média do ar provenientes de seis (6) estações meteorológicas do $8^{\circ}$ DISME/INMET (Distrito de Meteorologia do Instituto Nacional de Meteorologia) e da FEPAGRO/RS (Fundação Estadual de Pesquisa Agropecuária), distribuídas no entorno da APA do Ibirapuitã. 


\section{Resultados e discussão}

Para a biomassa aérea da vegetação medida em campo, foram encontrados valores médios de $1.829,36 \mathrm{~kg} /$ ha para o verão e $1.948,09 \mathrm{~kg} /$ ha para o inverno (Tabela 1). A faixa de valores considerada ótima por Neves et al. $(2009$, p. 1.690$)$ varia entre 2.000 a $2.500 \mathrm{~kg}$ de MS/ ha, para o mesmo protocolo experimental. Em estudo desenvolvido em uma área de vegetação do bioma Pampa, Barbieri $(2013$, p. 71) encontrou uma MF (massa de forragem) média de $3.057 \mathrm{~kg}$ de MS $/$ ha. Da mesma forma, Garagorry $(2012$, p. 81) obteve uma MF de $3.896 \mathrm{~kg}$ de MS/ha, considerados altos valores pelos mesmos autores.

0 perfil temporal com os valores médios das variáveis espectrais e da biomassa aérea da vegetação campestre demonstrou um comportamento-padrão entre a biomassa aérea e os índices EVI e NDVI e a banda espectral do NIR, com maiores valores no verão e menores no inverno, com exceção do ano de 2012,

Tabela 1 - Valores médios de biomassa aérea da vegetação campestre nos diferentes períodos do ano.

\begin{tabular}{c|c|c|c}
\hline \multirow{2}{*}{ Ano } & Estação & $\begin{array}{c}\text { Média de biomassa aérea (kg de } \\
\text { MS/ha) }\end{array}$ & Referência \\
\hline \multirow{2}{*}{2012} & Verão & $1.163,289$ & \\
& Inverno & $1.988,889$ & \multirow{2}{*}{$\begin{array}{c}2.000 \mathrm{a} \\
2.500 \mathrm{~kg} \text { de } \\
\text { MS/ha }\end{array}$} \\
\hline \multirow{2}{*}{2013} & Verão & $2.309,867$ & \\
\hline 2014 & Inverno & $1.907,289$ & \\
\hline
\end{tabular}

Fonte: Elaborado pela autora.

Figura 2 - Perfil temporal da biomassa aérea da vegetação e dados espectrais.

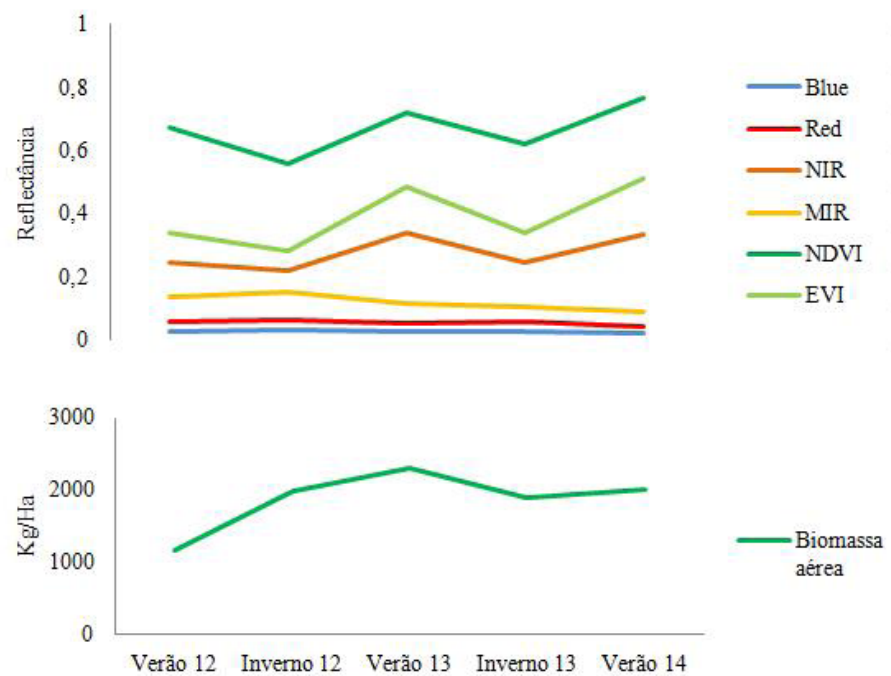

Fonte: Elaborado pela autora. 
em que a biomassa aérea da vegetação apresentou comportamento oposto (Figura 2).

$\mathrm{Na}$ época de maior crescimento/desenvolvimento da vegetação, que para a APA do Ibirapuitã inicia na primavera, e consequente aumento da atividade fotossintética e aumento das folhas verdes, ocorre uma diminuição da reflectância na região do visível e aumento no infravermelho próximo. Para o bioma Pampa, Bisso et

Figura 3 - Precipitação acumulada mensal e temperatura média do ar entre o período do verão do ano de 2012 e do verão de 2014 no entorno da APA do Ibirapuitã.

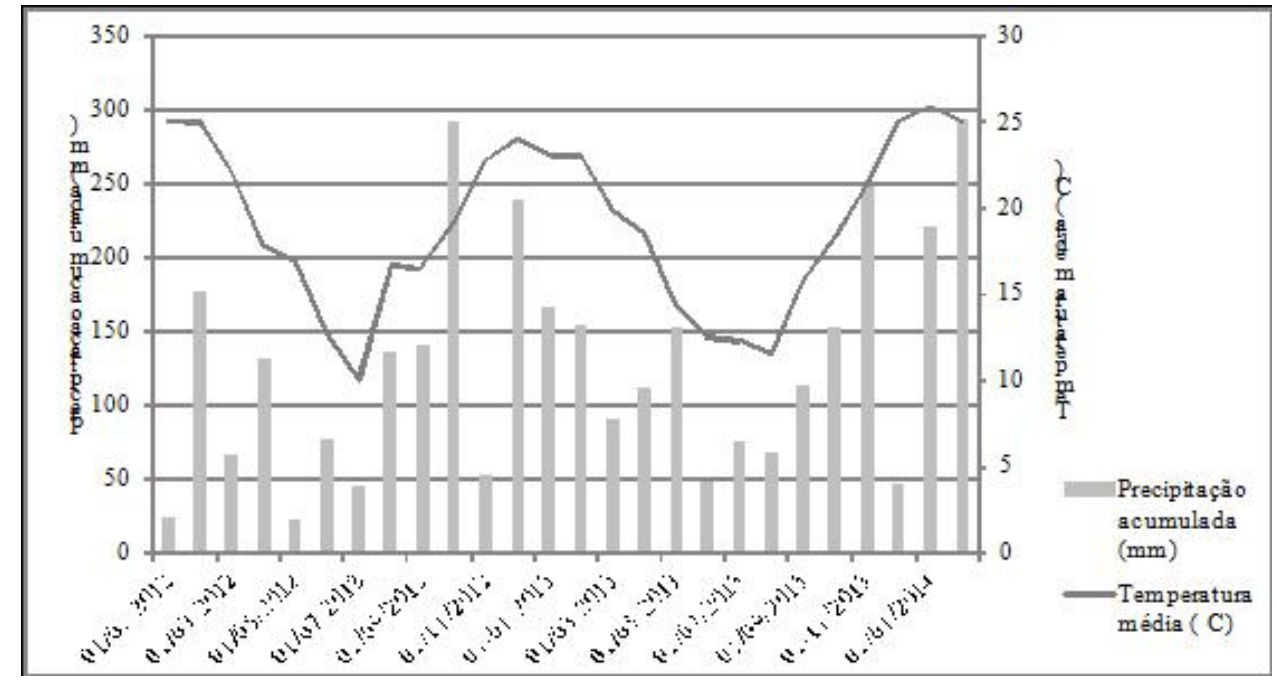

Fonte: Elaborado pela autora.

al. $(2010$, p. 3), observaram que os valores mais altos de NDVI ocorrem no período do verão e decaem no outono, atingindo os valores mais baixos no período do inverno.

O comportamento anômalo observado para a biomassa aérea no ano de 2012 (Figura 2) pode ser atribuído à variabilidade climática intra-anual relacionada principalmente à precipitação pluvial. $\mathrm{Na}$ Figura 3 observa-se que a precipitação no período de verão mostrou-se inferior quando comparado aos demais anos analisados nessa mesma estação, fato que pode ter ocasionado uma diminuição na biomassa.

Verificando a dinâmica sazonal de pastagem nativa em uma área do bioma Pampa e a sua relação com temperatura do ar e precipitação pluvial, Moreira et al. $(2013$, p. 53$)$ observaram que há maior correlação com a temperatura do ar (com maior intensidade após 30 dias de defasagem) e correlação fraca com dados de precipitação pluvial. Os autores destacam ainda que o NDVI permitiu a identificação da dinâmica fenológica e sazonal da vegetação campestre, com aumento nos valores durante as estações quentes do ano (primavera e verão) e diminuição nas estações mais frias (outono e inverno).

Da mesma forma, Bettolli et al. (2010, p. 256) avaliaram a relação entre a produtividade da pastagem e a variabilidade climática no Uruguai, confirmando quantitativamente a sensibilidade da vegetação à variabilidade do clima, sendo que a maior associação foi encontrada no outono (principalmente com as variáveis relacionadas à precipitação) e no verão.

No entanto, neste trabalho a análise de correlação entre o conjunto de dados de precipitação pluvial e biomassa aérea não retornou coeficientes significativos. Foram encontrados coeficientes de $r=-0,15$ para a precipitação acumulada de sete dias e $r=-0,06$ para 30 dias de defasagem.

0 coeficiente de correlação encontrado a partir da relação entre a disponibilidade de biomassa aérea da vegetação campestre com a resposta espectral detectada pelo sensor MODIS foi significativo (para $a=$ $1 \%)$ para as bandas do azul $(r=0,79)$, vermelho $(r=0,77)$, infravermelho próximo $(r=0,70)$, NDVI $(r=0,76)$ e EVI $(r$ $=0,91$ ). 0 resultado encontrado neste estudo é bastante semelhante ao trabalho desenvolvido por Zhao et al. (2014, p. 5.379), que obtiveram um $\mathrm{R} 2=0,75$ relacionando a biomassa de pastagem coletada em campo, na China, e o NDVI.

Relacionando a biomassa aérea da vegetação e o NDVI resultante do sensor Vegetation/SPOT, para o bioma Pampa, Fonseca et al. (2011, p. 39) encontraram um coeficiente de correlação de $r=0,517$, resultado inferior ao encontrado neste estudo.

A distribuição dos pontos nos gráficos (Figura 4) apresentou uma tendência linear e decrescente para 
as bandas do azul e do vermelho do sensor MODIS. A banda do MIR também apresentou tendência negativa. $\mathrm{Na}$ região do espectro visível, principalmente nos comprimentos de onda do azul e do vermelho, a radiação é utilizada pelas plantas no processo de fotossíntese, justificando a grande absorção de energia apresentada pelas folhas nesta faixa do espectro eletromagnético (Ponzoni; Shimabukuro, 2009, p. 18).

A banda espectral do infravermelho próximo (NIR) e os Índices de Vegetação EVI e NDVI apresentaram uma

Figura 4 - Gráfico de dispersão e análise de regressão demonstrando a relação entre a disponibilidade de biomassa aérea da vegetação campestre nativa medida em campo e a resposta espectral do sensor MODIS no bioma Pampa.
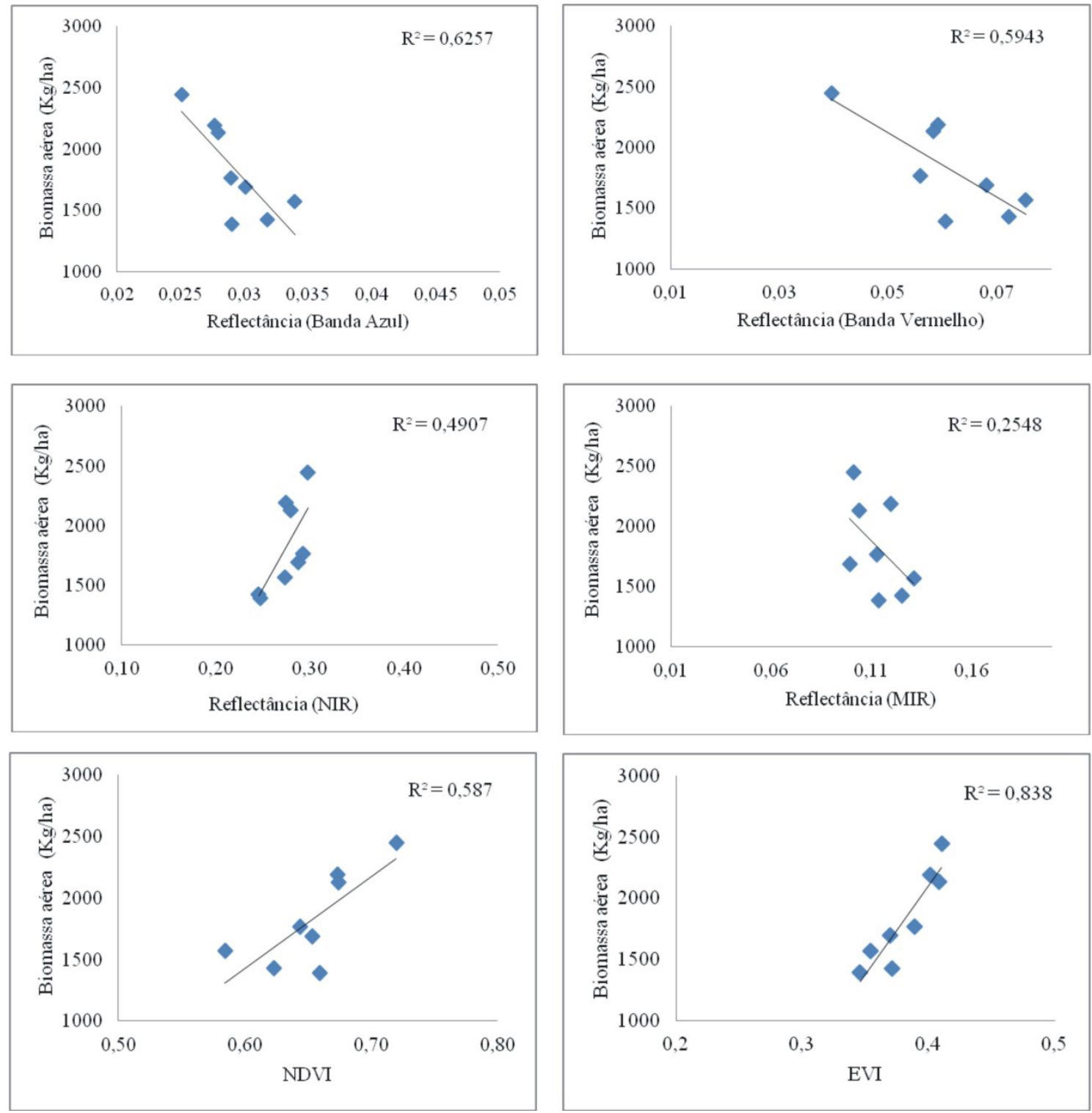

Fonte: Elaborado pela autora.

tendência linear e crescente entre os dados. Estudos demonstram que existe uma relação direta entre resposta no infravermelho próximo e variáveis relacionadas com biomassa. Além disso, há uma relação inversa entre a resposta no visível, particularmente no vermelho, e variáveis relacionadas com a biomassa das plantas, ou seja, à medida que um dossel vegetal se desenvolve, a sua reflectância aumenta no infravermelho próximo e absorve cada vez mais na região espectral do vermelho em função do aumento da fotossíntese (Jensen, 2009, p. 365). 


\begin{tabular}{ccc}
\hline Bandas espectrais & Modelo de regressão & $\mathbf{R}^{2}$ \\
\hline Azul & $\mathrm{y}=-113238 \mathrm{x}+5143,8$ & 0,63 \\
Vermelho & $\mathrm{y}=-26682 \mathrm{x}+3457,7$ & 0,59 \\
NDVI & $\mathrm{y}=7458,1 \mathrm{x}-3051,3$ & 0,58 \\
EVI & $\mathrm{y}=14391 \mathrm{x}-3655,5$ & 0,84 \\
\hline
\end{tabular}

Fonte: Elaborada pela autora.

No modelo apresentado para o coeficiente de determinação (significativo para $a=1 \%$ ), 63\% da variação observada na banda do azul, $59 \%$ na banda do vermelho, $58 \%$ para o NDVI e $84 \%$ para o EVI é explicada pelo fato de que a quantidade de biomassa aérea da vegetação também varia em diferentes períodos do ano, sendo observada uma relação de dependência entre a biomassa medida em campo e estimada por dados espectrais (Tabela 2).

\section{Conclusões}

A vegetação campestre nativa do bioma Pampa possui comportamento sazonal bem definido, com período de crescimento das espécies nos meses quentes do ano (primavera e verão), o que pode indicar que o comportamento padrão da biomassa aérea da vegetação no bioma Pampa tem relação principalmente com a temperatura média do ar.

No modelo apresentado, a relação entre a disponibilidade de biomassa aérea da vegetação e as bandas do azul, vermelho, infravermelho próximo, EVI e NDVI demonstraram que o aumento no valor da reflectância no NIR, NDVI e EVI é proporcional à quantidade de biomassa medida em campo. Porém, para as bandas do visível (azul e vermelho) esta relação é inversa: à medida que a biomassa aérea da vegetação aumenta, a reflectância nessas bandas diminui. 


\section{Referências}

BARBIERI, C. W. (2013). Utilização de dois intervalos de descanso entre pastoreios em pastagem natural com novilhas de corte na estação quente. Dissertação (Mestrado). Universidade Federal de Santa Maria, Santa Maria. $97 f$.

BETTOLLI, M. L. et al. (2010). Pastura natural de salto (Uruguay): relación con la variabilidad climática y análisis de contextos futuros de cambio climático. Revista Brasileira de Meteorologia, Rio de Janeiro, v. 25, n. 2, p. 248-259.

BISSO, A. B. A. et al. (2009). Análise da variação da resposta espectral da vegetação do bioma pampa, frente às variações da fenologia. In: XVI Congresso Brasileiro de Agrometeorologia (CBA), 16, 2009, Belo Horizonte. Anais... Belo Horizonte. Artigos. CD-ROM. p. 1-5.

BOLDRINI, I. I. (2009). A flora dos campos do Rio Grande do Sul. In: PILLAR, V. P.; MÜLLER, S. C.; CASTILHOS, Z. M. S.; JACQUES, A. V. A. (orgs.). Campos Sulinos: conservação e uso sustentável da biodiversidade. Brasília: MMA. Cap. 4, p. 63-77.

BOMBELLI, A. et al. (2009). An outlook on the Sub-Saharan Africa carbon balance. Biogeosciences, Göttingen, 6, p. 2.193-2.205.

BORK, E. W. et al. (1999). Rangeland cover component quantification using broad (TM) and narrow-band (1.4 nm) spectrometry. Journal of Range Management, Arizona, v. 52, n. 3, p. 249-257.

CARVALHO, P. C. F.; MARASCHIN, G. E.; NABINGER, C. (1998). Potencial produtivo do campo nativo do Rio Grande do Sul. In: PATIÑO, H. O. (ed.). Suplementação de ruminantes em pastejo. 1. Anais, Porto Alegre, p. 1-20.

FONSECA, E. L. et al. (2006). Eficiência de conversão da radiação fotossinteticamente ativa incidente em biomassa aérea da vegetação campestre natural no bioma Campos Sulinos do Brasil. Ciência Rural, Santa Maria, v. 36, n. 2, p. 656-659.

FONSECA, E. L. et al. (2011). Quantificação de biomassa acima do solo para Pradarias naturais no bioma dos Pampas. In: MAATHUIS, B. H. P.; MANNAERTS, C. M. (orgs.). GEONETCast - DevCoCast: Manual de Aplicações. Enschede: Faculty ITC, Enschede. p. 35-46.

GARAGORRY, F. C. (2012). Alternativas de manejo de pastagem natural submetida a pastoreio rotativo. Tese (Doutorado) - Programa de Pós-Graduação em Zootecnia, Universidade Federal de Santa Maria, Santa Maria. $210 f$.

HERINGER, I.; JACQUES, A. V. A. (2002). Acumulação de forragem e material morto em pastagem nativa sob distintas alternativas de manejo em relação às queimadas. Revista Brasileira de Zootecnia, Viçosa v. 31, p. 599-604.

JENSEN, J. R. (2009). Sensoriamento Remoto do Ambiente: uma perspectiva em recursos terrestres. São José dos Campos: Parêntese. 604 p.

MACHADO, L. A. Z. (1999). Manejo da pastagem nativa. Guaíba: Agropecuária. 158 p.

MENZEL, A. et al. (2001). Spatial and temporal variability of the phonological seasons in Germany from 1951 to 1996. Global Change Biology, Massachusetts, v. 7, p. 657-666.

MOREIRA, A. et al. (2013). Análise Combinada de Séries Temporais de NDVI, Temperatura do Ar e Precipitação Pluvial sobre Pastagem Nativa em Santa Maria, RS. Revista Ciência e Natura, Santa Maria, Edição Esp. p. 51-53.

NEVES, F. P. et al. (2009). Caracterização da estrutura da vegetação numa pastagem natural do Bioma Pampa submetida a diferentes estratégias de manejo da oferta de forragem. Revista Brasileira de Zootecnia, Brasília, v. 38, n. 9, p.1.685-1.694.

OVERBECK, G. E. et al. (2007). Brazil's neglected biome: The South Brazilian Campos. Perspectives in Plant Ecology, Evolution and Systematics, California, v. 9, n. 1, p. 101-116.

PARUELO, J. M. et al. (2000). Estimation of primary production of subhumid rangelands from remote sensing data. Applied Vegetation Science, Edinburgh, v. 3, p. 189-195. 
PONZONI, F. J.; SHIMABUKURO, Y. E. (2009). Sensoriamento Remoto no estudo da vegetação. São José dos Campos: Parêntese. 127 p.

SALMAN, A. K. D.; SOARES, J. P. G.; CANESIN, R. C. (2006). Métodos de amostragem para avaliação quantitativa de pastagens. Porto Velho: Embrapa Rondônia, Circular Técnica 84. 6 p.

SILVEIRA, V. C. P. et al. (2005). Qualidade da pastagem nativa obtida por diferentes métodos de amostragem e em diferentes solos na APA do Ibirapuitã, Brasil. Ciência Rural, Santa Maria, v. 35, n. 3, p. 582-588.

TUELLER, P. T. (2001). Remote sensing of range production and utilization. Journal of Range Management, Arizona, v. 54, n. 2, p. A77-A89.

WOLF, S. et al. (2011). Strong seasonal variations in net ecosystem CO2 exchange of a tropical pasture and afforestation in Panama. Agricultural and Forest Meteorology, Connecticut, v. 151, p. 1139-1151.

ZHAO, F. et al. (2014). Remote Sensing Estimates of Grassland Aboveground Biomass Based on MODIS Net Primary Productivity (NPP): A Case Study in the Xilingol Grassland of Northern China. Remote Sensing, Switzerland, n. 6. p. 5.368-5.386. 